\title{
HACIA LA IDENTIFICACIÓN TAXONÓMICA DE LAS REPRESENTACIONES DE PSITÁCIDOS EN LA CERÁMICA DE LA CUENCA DEL RÍO PARANÁ.
}

\author{
Flavia V. Ottalagano \\ CONICET; Facultad de Humanidades y Artes (UNR) flaviaott@yahoo.com.ar
}

Presentado: 03/05/2007 - Aceptado: 25/08/2008

\section{Resumen}

El objetivo de este trabajo es evaluar y proponer algunos criterios destinados a aportar datos con respecto a la identificación taxonómica de las aves psitaciformes presentes en la iconografía cerámica del Paraná medio. Como muestra de estudio se toma en este caso las representaciones de psitácidos recuperadas en siete sitios arqueológicos localizados en la margen entrerriana del río Paraná: Las Mulas I, El Largo 1, Arenal I y IV, Puerto Cuartel I y II, en el Dpto. La Paz; y La Palmera II, en el Dpto. Paraná.

Palabras claves: Paraná Medio, cerámica, identificación taxonómica, representaciones de psitácidos.

\section{Abstract}

The objective of this work is to evaluate and propose some criteria by which to provide data regarding the taxonomic status of psittaciform birds present in the iconography of middle Parana ceramic. In this case, the representations of parrot presents in the ceramic materials of the seven sites of this geographic area are analyzed: Las Mulas I, El Largo 1, Arenal I y IV, Puerto Cuartel I y II (Dpto. La Paz); and La Palmera II (Dpto. Paraná); which are located in the province of Entre Ríos.

Keywords: Middle Paraná, ceramic, taxonomical identification, representations of psittaciform birds.

\section{Presentación}

Este trabajo se inscribe dentro de un proyecto de investigación cuyo fin general es el estudio de las manifestaciones artísticas de los grupos de cazadores-recolectores-pescadores que poblaron las costas del río Paraná medio durante el Holoceno superior. Este registro artístico utiliza a los contenedores cerámicos como soporte y se compone -además de motivos abstractos incisos y pintados- fundamentalmente por representaciones de cabezas de animales, ejecutadas principalmente mediante modelado, y en menor medida por medio de trazos incisos. Si se consideran únicamente las representaciones ornitomorfas, las cuales son las más abundantes, las más habituales son las de aves psitaciformes, fácilmente distinguibles debido a los picos característicos de estos animales. En esta ocasión, se propone un abordaje de este registro, situando al análisis iconográfico como una vía para identificar 
las especies faunísticas que pudieron ser seleccionadas por estos grupos para ser expresadas en el arte alfarero.

\section{Antecedentes}

La visión Histórico-Cultural se constituyó como el planteo teórico más fuertemente arraigado en las investigaciones arqueológicas en las costas del Paraná, lo cual motivó que los aspectos artísticos fueran examinados mediante categorías difusionistas y como herramienta principal para identificar grupos culturales. Las representaciones zoomorfas y antropomorfas confeccionadas en cerámica fueron tomadas, de este modo, como el indicador más importante de la presencia de grupos culturales denominados Ribereños Plásticos o Goya-Malabrigo. La alfarería de tales grupos, y en especial el arte alfarero, fueron frecuentemente vinculados con aquellos propios de las poblaciones amazónicas (arawak), de las cuales habrían recibido sus principales influencias (ej. ver González 1947; Serrano 1946, 1961).

Sin embargo, más allá de estos fines clasificatorios, las figuras representativas fueron evaluadas bajo 3 visiones básicas: como piezas de carácter ritual, como piezas de cierta utilidad y/o con fines estéticos. Torres (1911) fue uno de los primeros autores en sostener la probable utilidad ceremonial de las mismas y su posible carácter totémico. La evaluación de distintas líneas de análisis (ej. registros arqueológicos, etnográficos y etnohistóricos) condujo a este autor a proponer vinculaciones entre tales representaciones y la creencia en espíritus tutelares, donde determinados animales vendrían a ser portadores de ciertas facultades sobrenaturales ${ }^{1}$. Contrariando estos argumentos, autores como Aparicio $(1936,1948)$ y Frenguelli (1927) consideraron que la iconografía cerámica habría sido utilizada en forma cotidiana como parte del ajuar doméstico ${ }^{2}$. Para ellos, estas figuras representativas pudieron funcionar como asas o bien como un complemento ornamental de los recipientes. De manera similar, para investigadores como Serrano $(1921,1946)$ y González $(1977)$ la preferencia por la representación de aves en el arte alfarero del Paraná no debió revestir carácter religioso, sino que esta predilección pudo responder más bien al hecho de modelar especies que eran comunes a la observación ${ }^{3}$; sosteniendo que las figuras plásticas serían interpretaciones indígenas de la naturaleza y de la fauna regional, es decir, un modo de reproducir el mundo cazador. De este modo, para estos investigadores, las piezas zoomorfas serían un medio fundamentalmente de expresión de valores estéticos, en orden de la satisfacción de un placer $\operatorname{artístico~}^{4}$.

\section{Punto de partida teórico}

El estudio del arte dentro del campo de la Arqueología ha ganado en los últimos años un nuevo ímpetu, en parte gracias al incremento de las investigaciones enfocadas en los aspectos simbólicos de las sociedades humanas pasadas (Morphy 1994). La preocupación por las expresiones artísticas prehispánicas ha retornado con posterioridad a la década de los '60, como resultado del énfasis en una arqueología del significado que centró sus objetivos no ya en la construcción de secuencias tipológicas de lo artístico, sino en sus roles activos y estratégicos dentro los grupos humanos involucrados.

Este trabajo pretende abordar la temática planteada partiendo de un concepto de cultura definido como un modo de organización de la experiencia, el cual da lugar a específicos 
ordenamientos simbólicos de las cosas (Foster 1994; Miller 1994; Renfrew 1994). En tal sentido, los objetos artísticos pueden ser concebidos desde una perspectiva estética y funcional, pero fundamentalmente representacional. El arte se entendería entonces como una representación simbólica de una realidad (Fiore 1996), se posicionaría como un modo de comprender el mundo en un particular contexto socio-cultural, y se desarrollaría poseyendo constreñimientos sociales (Hodder 1982).

Estos conceptos -el de cultura y el de arte- traen aparejada la idea que las sociedades humanas establecerían apropiaciones simbólicas de la naturaleza; constituyéndose esta última como una importante fuente de significados disponibles para las mismas (Foster 1994). El mundo natural, siguiendo a Lévi-Strauss (1997), propone al hombre un método de reflexión de sí mismos, en donde mundo social y mundo animal son pensados en términos recíprocos. Las formas animales, de esta manera, suministran un sustrato valioso para pensar la diferenciación de grupos e individuos, y constituyen un recurso metafórico básico para la expresión de las actitudes humanas. En tal sentido, ciertas cualidades propias, tanto de morfología como de comportamiento, tornan a muchas especies particularmente adecuadas para constituirse como fuente de significados (Douglas 1990; Tapper 1994, Saunders 1990; Tilley 1991).

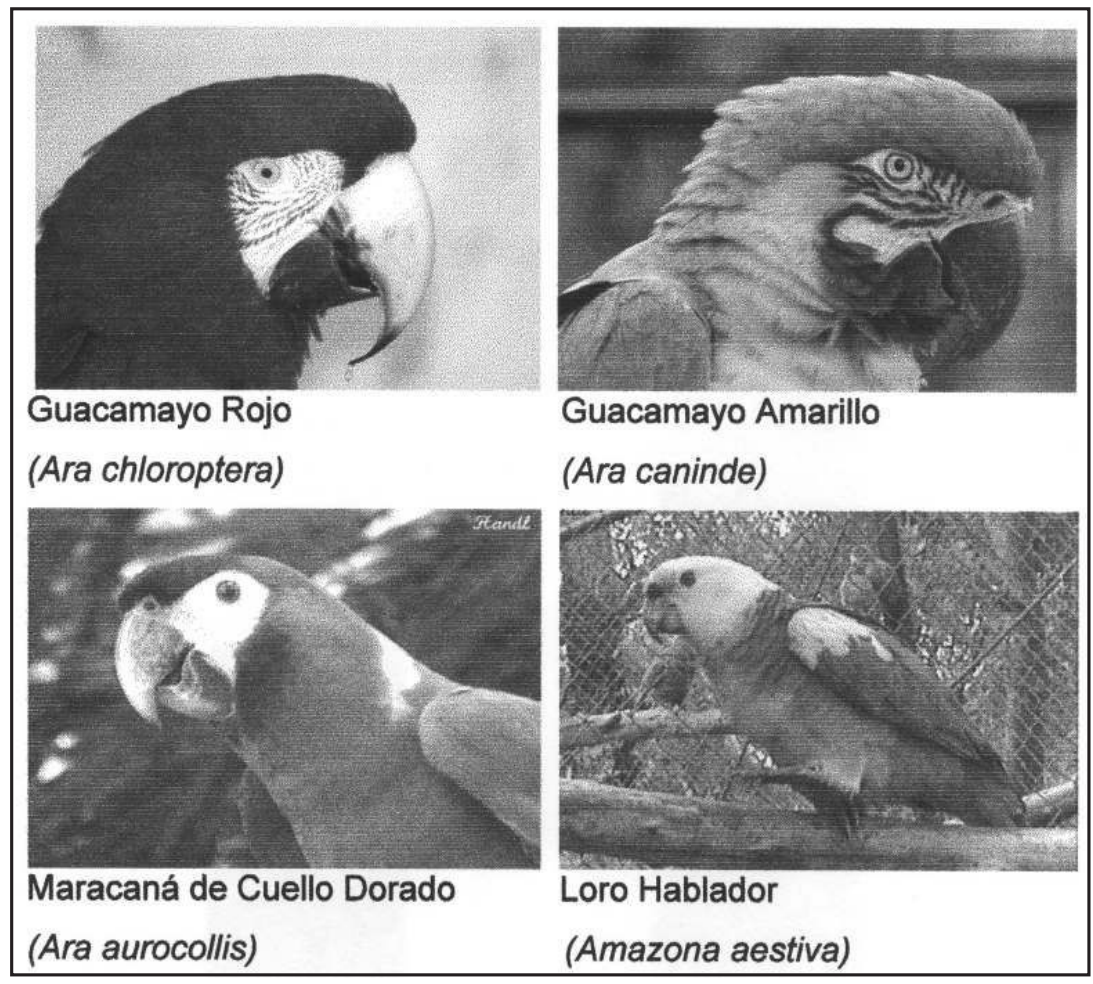

Figura 1. Ejemplos de aves psitaciformas sudamericanas (Fotos: Handl 2008; Spinuzza 2008). 


\section{Las aves psitaciformes sudamericanas}

Los psitácidos o loros se incluyen dentro del orden de los Psittaciformes, el cual abarca aproximadamente 327 especies que se agrupan mayoritariamente dentro de la familia Psittacidae La subfamilia Psittacinae absorbe la mayoría de los especimenes, de los cuales alrededor de unas 24 especies se encuentran presentes en territorio argentino en la actualidad (Narosky e Izurieta 2004). Los psitácidos sobresalen por su inteligencia, su domesticidad, y por la estructura especial de su legua, que en ciertos casos les posibilita reproducir sonidos semejantes a la voz humana; otorgándoles popularidad como mascotas (Hanzak 1968; Vigil 1973). La habilidades cognitivas avanzadas desarrolladas por estas aves permiten compararlas con el grupo de los primates, con los cuales comparten asimismo determinadas conductas sociales y biológicas (Iwaniuk et al. 2003).

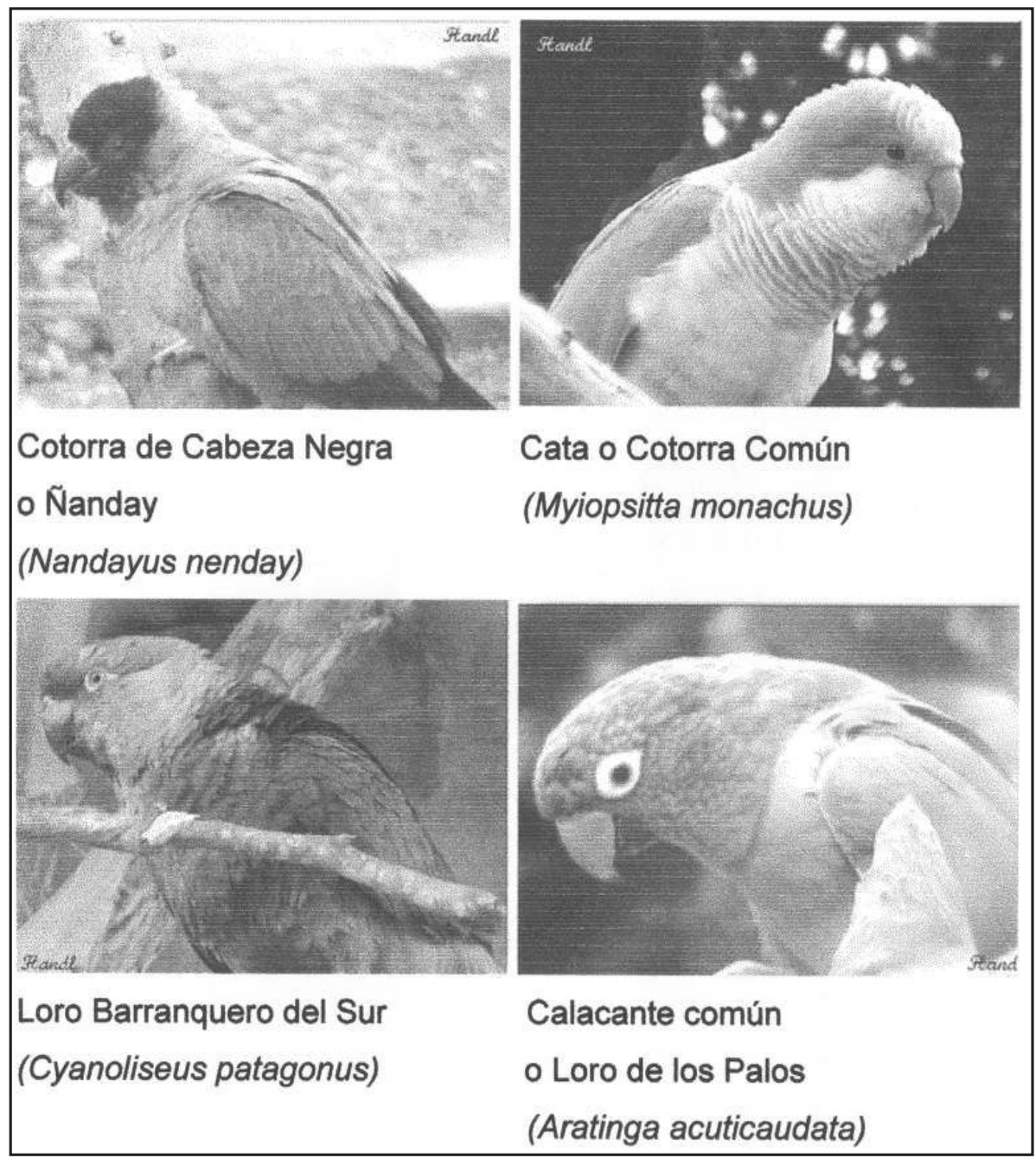

Figura 2. Ejemplos de aves psitaciformas sudamericanas (Fotos: Handl 2008). 
Un importante grupo de estas aves resultan originarias del Nuevo Mundo, y otras tantas típicas de Sudamérica. Como ejemplos de géneros sudamericanos de la subfamilia Psittacinae se pueden mencionar a los géneros Ara y Amazona (Figura 1), adaptados mayormente a climas tropicales y subtropicales; y aquellos distribuidos especialmente en áreas templadas o incluso frías, como ser los géneros Myiopsitta, Aratinga , Nandayus y Cyanoliseus (Figura 2). En el primer grupo se incluyen las distintas especies de los denominados guacamayos (género Ara), las cuales aparecen desde Centroamérica hasta el norte y nordeste de Argentina. A modo de ejemplo, el Maracaná de Dorso Rojo (Ara maracana), junto con el Maracaná de Cuello Dorado (Ara aurocollis) constituyen dos especies de guacamayos de pequeñas dimensiones que no superan los $40 \mathrm{~cm}$, cuyo hábitat comprende las selvas en galería y las zonas boscosas sudamericanas y del nordeste argentino. En el otro extremo, el Guacamayo Rojo (Ara chloroptera) es, por el contrario, una de los representantes más grandes, y también más vistosos de la familia. Alcanza a medir desde la punta del pico al extremo de la cola unos 78 a 90cm, y puede llegar a ser adiestrado para imitar vocablos humanos. Su vuelo se desarrolla a una relativamente corta distancia del suelo, y su grito característico que emite al amanecer y al atardecer, da origen a al nombre de su género. Se lo encuentra en zonas selváticas y en los grandes bosques desde Panamá y Colombia hasta Bolivia, Paraguay y nordeste de Argentina (ej. Chaco, Formosa y Misiones). El Guacamayo Amarillo (Ara caninde o Ara glaucogularis) presenta igualmente un porte elevado, aunque algo menor que el anterior, destacándose también por su gran colorido. Habita áreas de selvas en galería, e incursiona en bosques caducifolios. Principalmente se lo conoce en Bolivia, y aparentemente también en Brasil y Paraguay. Si bien, su presencia en la Argentina ha sido discutida se lo ha reportado en Salta, y existen datos de una probable presencia en las provincias de Misiones, Formosa y Chaco. El Guacamayo Verde (Ara militaris), uno de los ejemplares medianos (63 y $71 \mathrm{~cm})$, es conocido principalmente en Bolivia, llegando hasta las yungas del norte argentino. Finalmente, otro de los géneros adaptados a áreas de vegetación tropical y subtropical de Sudamérica y Argentina, es el Amazona, siendo el Loro Hablador (Amazona aestiva) una de sus especies más conocidas por su facilidad con que aprende a imitar palabras en estado de cautividad. Éste es más bien de porte pequeño, promediando los $36 \mathrm{~cm}$ de longitud, y presenta un plumaje de vivos colores en los que predomina el verde intenso y el amarillo, el cual alcanza la periferia de sus ojos. Forma bandadas de varios individuos y se extiende por el este de Bolivia, por Brasil y Paraguay, y por el nordeste de Argentina (ej. provincias de Misiones, Formosa, Salta, Corrientes, Entre Ríos, Chaco, y norte de Santa Fe) (Chebez 1994; Hanzak 1968; Narosky e Izurieta 2004; Narosky y Canevari 2004).

Por otro lado, entre aquellas especies que pueden encontrarse en áreas templadas y frías, se puede mencionar especialmente a las cotorras y catitas. La más abundante es la Cata o Cotorra Común (Myiopsitta monachus), un psitácido de $28 \mathrm{~cm}$ de color verde opaco, altamente sociable y el único que realiza nidos comunales (Narosky y Canevari 2004). Su distribución se extiende por las zonas de bosque y montes del sur de Brasil, Paraguay, Uruguay, y en Argentina, desde Río Negro a las provincias del norte. La Cotorra de Cabeza Negra o Nanday (Nandayus nenday), igualmente pequeña, es algo menos frecuente; apareciendo en territorio argentino por el área nordeste, llegando incluso la provincia de Bs. As. El Calacante común o Loro de los Palos (Aratinga acuticaudata) se desarrolla en regiones de selva en galería, boscosas y de monte de toda Sudamérica, y constituye otra especie de amplia distribución. Alcanza la mayoría de las provincias del centro y del norte argentino, llegando inclusive a zonas semi-áridas. Es de relativamente pequeño porte, como los dos casos anteriores, y su plumaje es fundamentalmente verde, en el que se destaca una coloración azulada a ambos 
lados de la cabeza. Por último, el Loro Barranquero del Sur (Cyanoliseus patagonus) es otro de los especimenes que forman parte de la avifauna de la Argentina en la actualidad. Presenta un plumaje de tinte oscuro, mide alrededor de $43-47 \mathrm{~cm}$ y se lo encuentra en áreas templadas y frías de Chile, sur de Uruguay, y en las provincias de San Juan, San Luís, Córdoba, Entre Ríos, Bs. As, La Pampa, Mendoza, Neuquén, Río Negro y Chubut (Hanzak 1968; Narosky e Izurieta 2004; Vigil 1973).

\section{Variabilidad taxonómica}

Resumiendo entonces, más allá de homogeneidad dada por su peculiar pico, la variabilidad dentro de la familia Psittacidae (subfamilia Psittacinae) se expresa en una serie de aspectos que trascienden la esfera morfológica. Entre los factores que introducen variabilidad dentro de este taxón se encuentran por ejemplo: variantes en los hábitos de comportamiento, anidación y cría; distintas capacidades para articular sonidos semejantes a la voz humana; diferentes habilidades cognitivas; $y$ adaptaciones a distintos ecosistemas. Si por el contrario se toma en consideración exclusivamente el aspecto físico de estos animales, entre los aspectos morfológicos que introducen variabilidad pueden considerarse principalmente: a) las dimensiones corporales; b) el color del plumaje; c) la morfología de los picos; y d) el área facial carente de plumas, presente en los ejemplares de guacamayos y ausente en los demás géneros.

Los distintos géneros e inclusive las distintas especies de la familia Psittacidae comprenden específicos colores, o específicas combinaciones de colores; siendo sus tamaños variables entre los escasos centímetros y casi el metro de longitud. Por otro lado, la morfología del pico, en cuanto a su longitud respecto del tamaño de la cabeza de los animales, puede ser tomada como un rasgo variable entre los distintos géneros de la familia. Así por ejemplo, las especies fundamentalmente del género Amazona y Ara presentan picos generalmente más pronunciados en los que se destaca una mayor proyección de la mandíbula superior sobre la inferior, en comparación, por ejemplo, con la mayoría de las especies de cotorras y calacantes. Por último, todas las especies de guacamayos presentan la particularidad de ostentar en la periferia de los ojos y sobre las mejillas una zona carente de plumas o signada por la presencia de hileras de pequeñas plumas muy esparcidas. Este atributo peculiar es compartido tanto por los guacamayos medianos y grandes, como por los más pequeños, conocidos como Maracanás (Hanzak 1968; Vigil 1973).

\section{Aspectos metodológicos: bajada al registro artístico.}

Si considera exclusivamente las características físicas de las aves psitaciformes, y se descarta aquellos aspectos que introducen variabilidad pero que trascienden la esfera morfológica, los indicadores taxonómicos más evidentes para marcar diferencias de género dentro de la familia Psittacidae serían en primera instancia: el color del plumaje y las dimensiones corporales. Tales características mencionadas, aunque detectables visualmente, no pueden sin embargo ser observadas en el registro iconográfico por dos razones fundamentales: a) las escalas de las representaciones zoomorfas se correlacionan proporcionalmente con las dimensiones de los contenedores cerámicos de los que forman parte, y no con los tamaños reales de los animales, y b) la coloración de los ejemplares reproducidos tampoco posee correspondencia con la realidad, ya que en el caso de estar 
pintados los únicos colores utilizados fueron el rojo y, en menor proporción, el blanco. Por otra parte, el largo de los picos de los psitácidos, si bien puede ser también tomado como un indicador taxonómico, resulta un atributo de gran fragilidad en el registro artístico cerámico; motivo por cual en un elevado numero de ejemplares se presenta fracturado. A estas limitaciones debe agregarse otras, como ser el hecho de que se ha modelado casi con exclusividad la cabeza del animal; razón por la que los elementos disponibles en el momento de intentar una identificación taxonómica más precisa sobre el registro iconográfico se ven acotados.

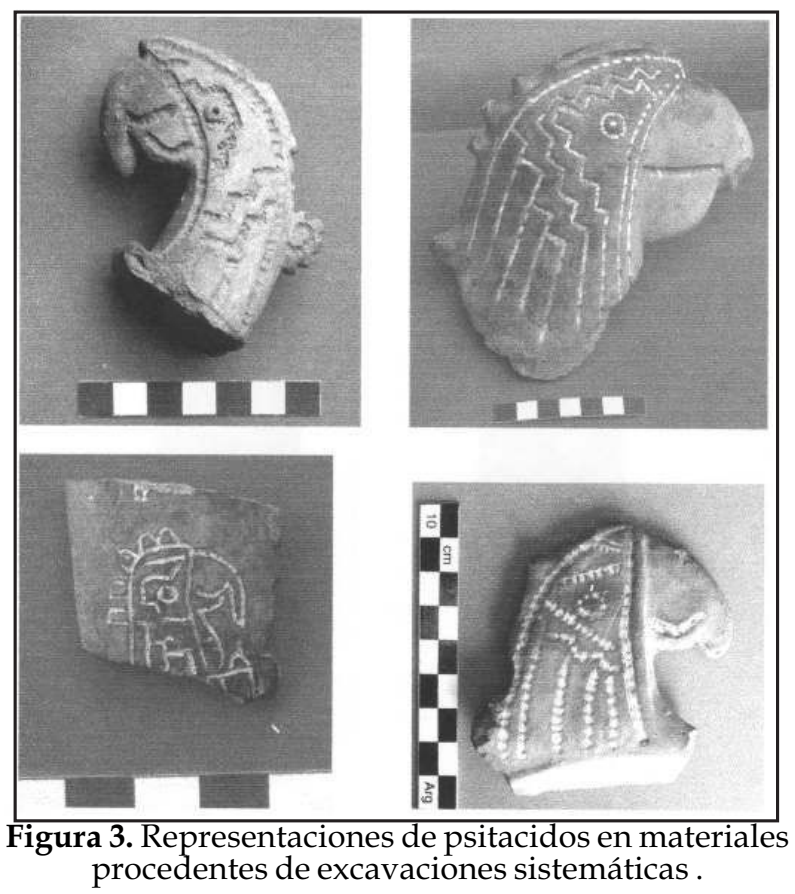

El área facial carente de plumas, atributo exclusivo de las especies del género Ara, es tal vez, entonces, uno de los pocos caracteres que podrían tenerse en cuenta para evaluar identificaciones taxonómicas en el registro artístico. Este trabajo parte de la idea, por consiguiente, que esta área desnuda podría llegar a encontrar un correlato arqueológico en ciertos trazos incisos que con frecuencia tienden a demarcar un sector alrededor de los ojos de las aves representadas en la iconografía cerámica del área del Paraná5 (Figura 3).

\section{Pasos metodológicos}

1. Se procedió primeramente a dividir la muestra de representaciones de aves psitaciformes de la iconografía cerámica de los sitios considerados en dos conjuntos, de acuerdo al acompañamiento o no de detalles realizados mediante técnicas de incisión: a) representaciones lisas b) representaciones con detalles incisos (Figura 4).

2. Al grupo de representaciones con detalles incisos, a su vez, se lo discriminó según la aparición o la ausencia de líneas incisas que sugirieran la intención de demarcar una zona en la periferia de los ojos de las aves (Figura 3 y 4 ). 


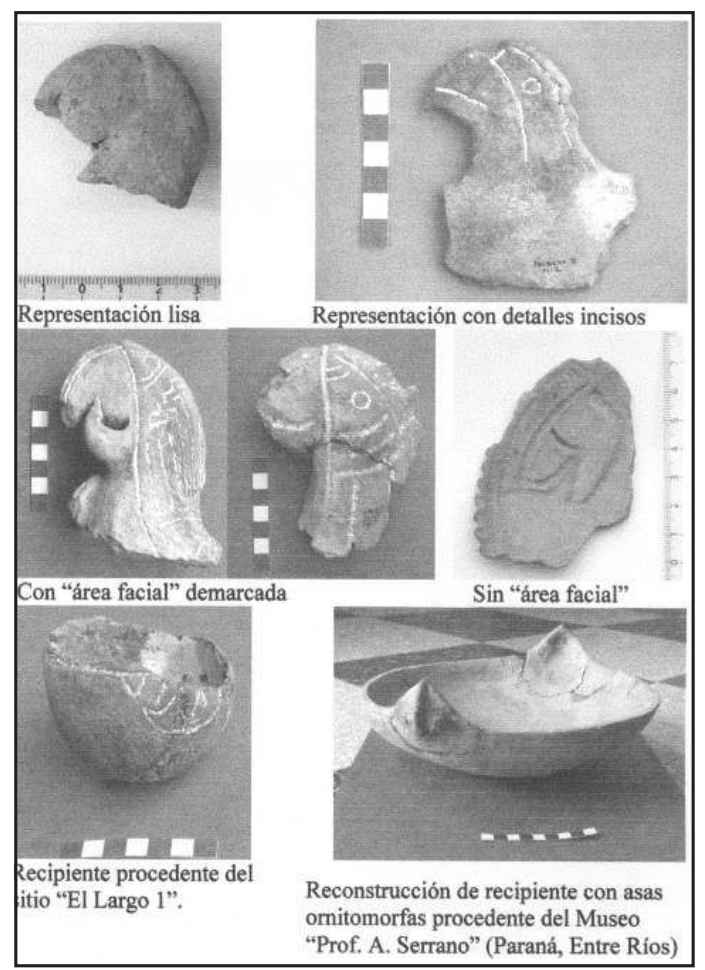

Figura 4. Representaciones de psitacidos en materiales de excavación y ejemplos de su ubicación en los recipientes.

3. Se tuvieron en cuenta la profundidad de aparición de las figuras en la estratigrafía de los sitios, así como sus áreas contextuales correspondientes. Para aquellas representaciones procedentes de excavaciones sistemáticas, y puesto que se contaba con información de campo precisa, su asignación a cada uno de estos grupos se registró teniendo en cuenta los niveles artificiales en que ellas aparecían. Luego fueron contabilizadas junto con aquellas extraídas durante las primeras intervenciones arqueológicas a fin de extraer porcentajes totales ${ }^{6}$. Igualmente con los materiales de excavación, se registró la localización de las figuras de acuerdo a sus áreas contextuales. Particularmente se tuvo en cuenta la relación de las mismas con los sectores de entierro.

Como consecuencia de la imposibilidad de localizar algunos de los ejemplares recuperados durante estas primeras intervenciones, el análisis de los atributos incisos de los animales debió ser complementado con la información publicada en fotos (publicadas en Serrano 1946). Debido a que en algunos casos este cuerpo de información no permitió un examen seguro de los detalles incisos de las figuras, se englobó a estos casos bajo el término de indeterminados. Igualmente fueron clasificadas como indeterminadas aquellas figuras que por su fragmentación, no fue posible asignarlas a ninguno de los dos últimos grupos.

La muestra total analizada contó con 97 representaciones de aves psitaciformes, las cuales forman parte de los materiales de superficie y de excavación de 7 sitios arqueológicos 


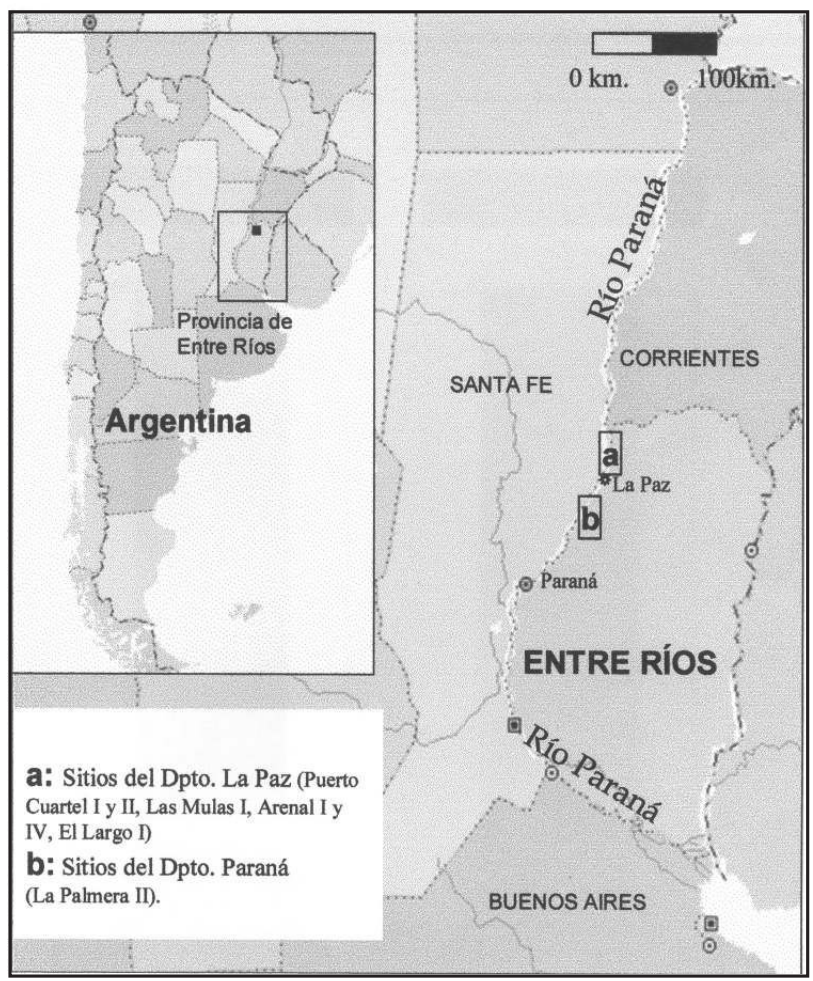

Figura 5. Localización de los sitios arqueológicos considerados

localizados en la margen entrerriana del Paraná medio (Figura 5); materiales depositados actualmente en el Museo "Prof. A. Serrano" de la ciudad de Paraná, Provincia de Entre Ríos.

\section{Contexto del registro artístico.}

La presencia de representaciones de figuras animales y humanas en artefactos cerámicos se registra por un amplio sector del área Nordeste de la República Argentina (Ceruti 1999, 2000). Este arte mobiliar cerámico se asocia a ocupaciones cazadoras-recolectoras-pescadoras del Holoceno Superior; grupos específicamente adaptados al ámbito fluvial del Paraná. Si bien algunos fechados sugieren la presencia de estas poblaciones unos 2.000 años atrás, su desarrollo más importante, sin embargo, habría tenido lugar a partir de los 1.000 años AP aproximadamente. A partir de entonces y hasta momentos cercanos a la conquista española, estos grupos habrían intensificado su presencia sobre las costas e islas de la llanura aluvial del Paraná medio principalmente, alcanzando el delta y remontando el río Uruguay (Ceruti 2000, 2004). En lo que respecta a las áreas próximas al río Paraná, tales manifestaciones artísticas mantienen como límites aproximados de registro: al norte, las ciudades de Corrientes y Resistencia, en las Provincias de Corrientes del Chaco respectivamente; al sur, la desembocadura en río de La Plata del brazo más meridional del Paraná, esto es, el Paraná de las Palmas; al oeste, el río Salado; y al este, el límite dado por la culminación de la terraza del Paraná y el comienzo de las tierras altas de Entre Ríos (Ceruti 1999, 2000). 
Los contenedores cerámicos

Los contenedores cerámicos asociados a los grupos del ambiente fluvial del Paraná presentan un grado de fragmentación intenso, con escasa presencia de ejemplares enteros o con posibilidades de una reconstrucción completa. No obstante, expresan por lo general formas globulares con base redondeada, contornos simples y con la presencia de orificios de suspensión y asas. Si bien resulta más frecuente el hallazgo de recipientes lisos que no presentan ninguna clase de decoración, los contenedores de alfarería constituyen prácticamente el único soporte conocido para el registro artístico de los grupos de cazadoresrecolectores del área del Paraná.

Este registro artístico se encuentra conformado por motivos abstractos realizados mediante incisión o pintura, y fundamentalmente por motivos representativos elaborados mediante modelado y, en menor proporción, mediante técnicas incisas. Estos últimos presentan un carácter predominantemente zoomorfo, y en algunos casos antropomorfo; tratándose pocas veces de representaciones de animales completos, sino más bien de cabezas dispuestas a modo de asas o apéndices incorporadas a contenedores lisos, pintados ${ }^{7}$, con guardas incisas abstractas, así como a alfarerías gruesas sin fondo (Figura 4). La iconografía de psitácidos constituye entre el 36\% y el 95\% del total de representaciones zoomorfas y antropomorfas de los sitios considerados, entre las que se incluyen principalmente otros órdenes de aves (ej. falconiformes, estrigiformes), además de reptiles (ej. quelonios, ofidios) y mamíferos (ej. felinos, primates).

Tanto los recipientes decorados como aquellos lisos aparecen en contextos domésticos y mortuorios; presentándose, en algunos casos, como un claro acompañamiento funerario. Las elecciones tecnológicas implementadas en uno y otro caso (recipientes cerámicos decorados y no decorados) no parecen diferir substancialmente; destacándose generalmente la presencia de superficies bien alisadas, con una pasta compacta, gris y con tiesto molido (Ottalagano 2005, 2007a, 2007b, 2007c).

Los sitios considerados

Se tomaron 7 sitios localizados en la margen izquierda del Paraná, correspondiente a la Provincia de Entre Ríos (Figura 5). Casi la totalidad de los mismos se sitúa en el Dpto. La Paz (Terraza del Paraná), a una distancia aproximada de entre 10 y $37 \mathrm{~km}$ al noreste de la ciudad de La Paz; manteniendo una separación mutua de escasos metros y los $27 \mathrm{~km}$. De norte a sur estos sitios son: Puerto Cuartel I (cuenca del Río Guayquiraró); Puerto Cuartel II; Las Mulas I (cuenca del Arroyo Las Mulas); Arroyo Largo I (Llanura de inundación del Paraná, Isla Curuzú Chalí); Arroyo Arenal I y IV (Cuenca del Arroyo Arenal). En tanto que el sitio La Palmera II es el único que se sitúa en el Dpto. Paraná (Terraza del Paraná, Cuenca del Arroyo Hernandarias), a una distancia aproximada de unos $73 \mathrm{~km}$ del resto de los sitios.

Las primeras intervenciones arqueológicas en Las Mulas 1 y El Largo 1, fueron efectuadas en la década del '40 a cargo de investigadores como Serrano (ver ej. Serrano 1946). Posteriormente, los trabajos se continuaron en ambos sitios hacia fines de los '70 y la década de los '80; llevándose adelante excavaciones estratigráficas por parte de Ceruti, quien intervino asimismo los demás sitios considerados, durante este período y comienzos de los '90 (ver ej. Ceruti 1980, 1989, 1999). Él énfasis colocado en este conjunto de sitios arqueológicos para 
abordar el arte mobiliar cerámico de los grupos cazadores-recolectores del Paraná Medio, radicó por consiguiente, en que éstos otorgaban un cuerpo de información contextual considerable para el tratamiento de esta temática en el sector entrerriano. Ya que, a excepción de A. Arenal IV y Puerto Cuartel II, el resto cuenta con excavaciones estratigráficas practicadas.

Además de los fragmentos de alfarería, los cuales constituyen los materiales más abundantes del registro arqueológico de tales sitios (en el que predominan los tiestos lisos por sobre los decorados mediante técnicas de incisión, pintura o modelado), se encuentran ítems líticos, arqueofauna y enterratorios. El material lítico suele ser escaso, destacándose la presencia de algunos artefactos (ej. arpones, puntas de proyectil, artefactos de molienda, lascas). Las Mulas I, La Palmera II, Arenal I y Puerto Cuartel I registran enterratorios de diverso tipo, con o sin acompañamiento funerario, entre los que se incluyen: entierros primarios extendidos (decúbito dorsal y ventral), entierros secundarios de paquetes funerarios de individuos de distintas edades, y entierro de piezas óseas aisladas (ej. La Palmera II); además de numerosos restos humanos dispersos (ver ej. Ceruti 1989).

Se cuenta con tres fechados radiocarbónicos para los mismos. Uno de ellos es para Las Mulas I: 950 ( \pm 120$)$ AP (INGEIS, carbón), profundidad 0,70-0,80m. Según este fechado, este sitio desarrollaría una ocupación de entre los $1450 \mathrm{AP}$ y momentos cercanos a la conquista española (Ceruti 1989). En tanto que El Largo I sostendrían una antigüedad de 1380 (+-100) AP (INGEIS, carbón) a los 0.90-1 m de profundidad, en concordancia con su ocupación más antigua; y una antigüedad de 900 (+-100) AP (INGEIS, carbón) a los 0,60-0,70 m de profundidad (Ceruti 1999).

\section{Contexto ambiental y faunístico}

El ambiente actual en el área del Paraná medio, y en el cual se insertan los sitios arqueológicos considerados, se ubica dentro de la Región Neotropical, Subregión GuayanoBrasileña (Ringuelet 1961). Se asocia a un clima correspondiente a latitudes medias, el cual puede ser considerado como subtropical húmedo, entendido como básicamente templado y lluvioso, con veranos calurosos. Este clima se relaciona genéricamente a formaciones de bosque lluvioso templado o tropical, pudiéndose desarrollar también formaciones transicionales como en el caso de las praderas (Strahler 1975). Las formaciones de selva y bosque en galerías, que constituyen fajas estrechas $(50 \mathrm{~m}-200 \mathrm{~m})$ de formaciones vegetales cerradas, recorren las márgenes del cauce del río Paraná y de algunos de sus afluentes. Hasta aproximadamente la ciudad de Corrientes la galería paranaense puede ser considerada prácticamente como una prolongación de selva misionera. Más allá de este punto las formaciones arbóreas subtropicales comienzan a experimentar una paulatina reducción; situación intensificada más aún como resultado de la creciente antropización del área, la cual ha derivado en intensas modificaciones ecológicas y geomorfológicas (Campos 2001; Ceruti 1991; Frenguelli 1941; Manzi y Premoli 1973; Parodi 1964).

Si bien la escasez de datos para él área no posibilita delinear un esquema muy preciso del desarrollo paleoambiental de la región (Tonni 2004), se estima que para el Holoceno Tardío las condiciones climáticas áridas que predominaron desde los $3.500 \mathrm{AP}$, dejaron paso a condiciones de mayor temperatura y humedad hacia aproximadamente los 1150-750AP, lapso comprendido por el evento climático conocido como Máximo Térmico Medieval ${ }^{8}$ (Ceruti 1988, Deschamps et. al. 2003; Iriondo 1991). Tales circunstancias habrían permitido el resurgimiento de las formaciones selváticas de Misiones y de la galería paranaense; 
experimentando las especies de fauna pampásica un lento retroceso, y las basílicas un progresivo avance hacia áreas más meridionales de esta galería, proceso que habría favorecido la convivencia circunstancial de elementos faunísticos de ambos ambientes (Ceruti 1988).

Los restos zooarqueológicos asociados a las poblaciones ribereñas indican fundamentalmente elementos faunísticos semejantes a los que existen en el presente en la zona. Esto es, mayoritariamente peces -ej. bagres armados (Doradidae)-, aves -ej. biguá (Phalacrocórax brasilianus) y patos (Anatidae)-, moluscos (ej. Diplodon y Ampullaria) y mamíferos acuáticos, entre los que se encuentran particularmente nutrias (Myocastor coypus), ciervo de los pantanos (Blastocerus dichotoms) y viracho (Mazama cf. gouazoubira); además de otros mamíferos como el yaguareté (Felis onca) y el carpincho (Hydrochaeris hydrochaeris) (Ceruti 1988; Iriondo 1991; Salemme et. al. 1987).

Los conjuntos zooarqueológicos se caracterizan por presentar por lo general una alta fragmentación y una la alta frecuencia de elementos poco diagnósticos desde el punto de vista de la identificación de especies (Ottalagano 2007a). Esto posiblemente sea el resultado de procesos tafonómicos y post-depositacionales sobre restos faunísticos de medianas a pequeñas dimensiones, más que una consecuencia de la acción atrópica en sí misma (Salemme et. al.1987). Si bien para la mayoría de los sitios considerados en este trabajo no se cuenta con un registro específico correspondiente a su avifauna, en el caso del sitio Arenal 1 las especies de ave que se registran entre sus materiales de estratigrafía están fundamentalmente vinculadas a ambientes acuáticos fluviales. Entre ellas se destacan: orden Tinamiformes (familia Tinamidae); orden Pelecaniformes (familia Phalacrocoracidae); orden Ardeiformes (familia Ciconidae; orden Anseriformes (familia Anatidae); y orden Ralliformes (familia Rallidae) (Tonni et al. 1985).

\section{Resultados}

Se computaron un total de 97 representaciones de psitácidos, recuperadas entre las primeras intervenciones arqueológicas efectuadas durante la década de 1940 y las excavaciones más recientes, realizadas hacia fines de $\operatorname{los}^{\prime} 70$ y principios de $l o s ' 90$. Proceden mayoritariamente del sitios Las Mulas I, en el que se registraron 54 ejemplares; y en menor medida, de los sitios El Largo I (19 ejemplares), Arenal I (10 ejemplares), La Palmera II (6 ejemplares), Puerto Cuartel I (4 ejemplares), Puerto Cuartel II y Arenal IV (2 ejemplares cada uno). Las representaciones de psitácidos, junto con los fragmentos de representaciones de probables psitácidos, conforman un promedio de $73 \%$ del total de las representaciones ornitomorfas de los sitios, y el $48 \%$ con respecto a la totalidad de las representaciones zoomorfas registradas en los mismos.

Del total de las representaciones de psitácidos recuperadas en los 7 sitios arqueológicos, el 84,5\% (82 ejemplares) presentó detalles decorativos realizados mediante técnicas de incisión (surco rítmico principalmente, y en menor medida, incisión de línea y de punto); siendo este porcentaje, por consiguiente, el que expresa la muestra factible de analizarse mediante esta propuesta de análisis iconográfico. El 15,5\% restante (15 ejemplares), estuvo integrado por representaciones modeladas totalmente lisas (Figura 6).

Dentro del grupo conformado por representaciones de aves psitaciformes con detalles incisos, el 60\% (49 ejemplares) advirtió la presencia de líneas, ya sea rectas o escalonadas, destinadas a resaltar un área alrededor de los ojos y sobre las mejillas de las aves ejecutadas (Figura3). Un 10 


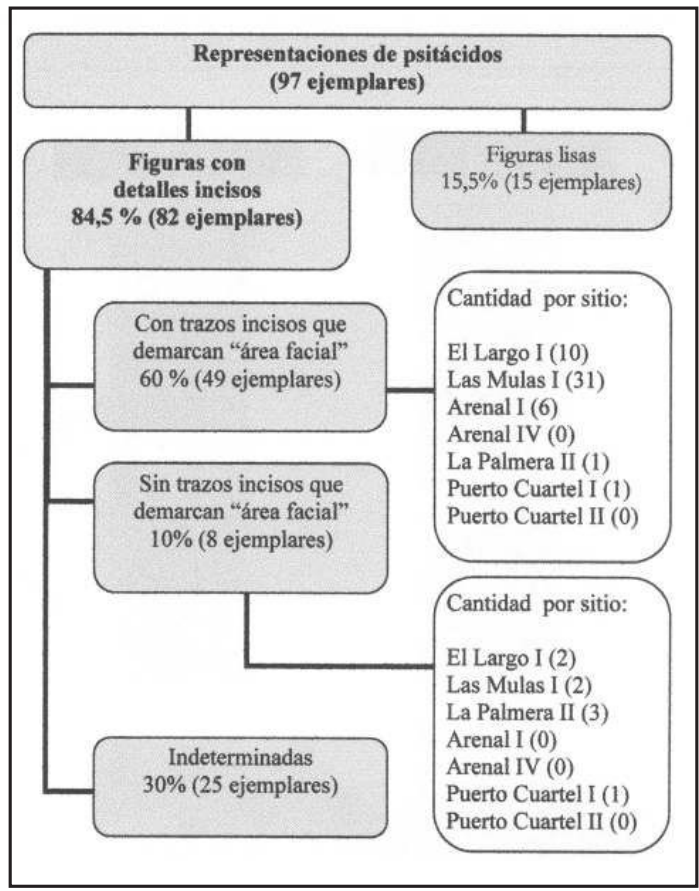

Figura 6. Esquema de Resultados obtenidos

\% de este conjunto (8 ejemplares) se encontró decorado con detalles incisos sin aparentemente implicar la intención de otorgar visibilidad a este sector de la cara de las aves diseñadas. Mientras que un porcentaje considerable (30\%,25 ejemplares) fue asignado a las figuras de loros decoradas mediante incisión, a las cuales sin embargo, no fue posible la identificación de este atributo.

Las representaciones de psitácidos que manifiestan la demarcación de un sector periférico alrededor de sus ojos, comienzan a registrarse en el sitio Las Mulas I a partir de los 0,70-0,80 m de profundidad, nivel fechado en $950 \pm 120$ a.p. (INGEIS, carbón) ${ }^{9}$; mientras que para el caso de El Largo I, este tipo de representaciones aparecen a nivel superficial en el área de ocupación más reciente. Para el resto de los sitios excavados, si bien no se poseen fechados absolutos, los datos indican la presencia de las mismos entre los 0,50 y los 0,20 m de profundidad en Arenal I; entre los 0,20 y los 0,10 m en el caso de La Palmera II; y entre los materiales de superficie, en el caso de Puerto Cuartel I.

Las figuras de psitácidos que expresan una "área facial" demarcada aparecen en la estratigrafía de los sitios en distintos contextos de asociación. De este modo, computando solamente aquellas figuras en las que se ha podido contabilizar este atributo facial por no encontrarse fracturadas, la disposición de las mismas en relación a los entierros se registra del modo siguiente:

Arenal I: $17 \%$ en asociación directa con los enterratorios; $66 \%$ en asociación probable con los mismos; y el 17\% en sectores sin entierros.

La Palmera II: $100 \%$ en asociación con áreas de entierro.

Puerto Cuartel I: 100\% en sectores sin entierros (materiales de superficie). 
Las Mulas I: $14 \%$ en asociación directa con los enterratorios; $28,5 \%$ en asociación con áreas de entierro; $36 \%$ en asociación con restos humanos aislados, $21,5 \%$ en sectores sin entierros.

\section{Consideraciones finales}

La representación de aves psitaciformes en los contenedores de cerámica constituye un registro artístico frecuente de los grupos cazadores-recolectores-pescadores que poblaron durante el Holoceno Superior un amplio sector ribereño del Nordeste argentino. De todas las representaciones ornitomorfas, aquellas correspondientes a psitácidas resultan, quizás, las más distinguibles debido sus característicos picos curvos y ganchudos. Sin embargo, de los distintos atributos que permiten discriminar entre sí a los diferentes géneros de la familia Psittacidae, se observa que son muy acotados los rasgos efectivos que pueden ser contabilizados para llevar adelante una identificación taxonómica precisa sobre la base del registro iconográfico. Siguiendo esto, se consideró en este trabajo que la aplicación de las técnicas de incisión, más que con una intención decorativa en sí misma, pudo haber estado destinada a completar detalles reales de los animales representados en el arte; y por consiguiente, orientada a destacar visualmente atributos físicos y particulares de las especies. De este modo, por ejemplo, las áreas faciales desnudas propias de las especies de Aras podrían encontrar correlato en la iconografía cerámica en ciertos trazos incisos que con frecuencia tienden a demarcar un sector alrededor de los ojos de las aves representadas. Algunas otras posibilidades más, sin embargo, pueden ser incluidas en la interpretación de los diseños incisos de los psitácidos de cerámica, como ser el propósito de diferenciar áreas con distintas tonalidades en la cara de otros géneros y especies de la familia; tal como podría ser el caso de la Amazona aestiva o Loro Hablador, en el que parte de su plumaje amarillo, como se mencionó, llega a extenderse derredor de los ojos de esta ave.

Dado que el género Amazona, y más particularmente el Ara, constituyen taxones especialmente adaptados a áreas de vegetación tropical y subtropical (ej. selvas, selvas en galería y zonas boscosas); es posible que las poblaciones humanas pasadas hayan otorgado a estas especies una importancia adicional por el hecho de no participar de la fauna local inmediata. Debe acotarse, no obstante, que varias de las especies más llamativas del género Ara se encuentran en la actualidad extintas, reducidas en población o bien acotadas a sectores más al norte que su hábitat habitual, debido a un conjunto de factores entre los que se incluyen la deforestación, la contaminación y la captura indiscriminada. La galería paranaense, la cual tiende a actuar como un corredor de especies, se ha visto afectada en los últimos años por la actividad antrópica en general, la cual a contribuido a mermarla considerablemente, acarreando concomitantes modificaciones ecológicas (Chebez 1994; Manzi y Premoli 1973). De este modo, por ejemplo, especies como el Ara Chloroptera, una de los ejemplares más llamativos de guacamayos, ha sido descrito por cronistas del siglo XIX al igual que con respecto a otros guacamayos extinguidos en el presente- en provincias como el Chaco y Corrientes; mientras que hoy se lo encuentra circunscrito fundamentalmente a las cercanías de la selva misionera (Chebez 1994). Por otro lado, si se acepta el establecimiento de condiciones de mayor humedad y temperatura para el área hacia el 1150-750 AP (Ceruti 1988) -las cuales serían concordantes con una parte de la ocupación de los sitios-, es probable también que el avance de estas condiciones ecológicas en zonas más australes de las que hoy se presentan en la actualidad haya podido extender, en alguna medida, el hábitat natural de estas especies. 
Uno de los lineamientos teóricos principales tomados como guía en los argumentos de este trabajo fue el considerar que en el arte participan componentes del simbolismo de los grupos humanos pasados (Hodder1982), y que la naturaleza se conforma como una fuente de significados disponibles para las sociedades (Foster 1994). En las poblaciones cazadorasrecolectoras, particularmente, el entorno se presenta, más aún, como un medio a la vez natural y artificial; constituyéndose como una naturaleza culturalmente percibida (Ingold 1993, 1996). Bajo la luz de estas perspectivas, las cuestiones de significado y aquellas relacionadas con el por qué ciertos objetos son utilizados para significar, constituyen aspectos no totalmente arbitrarios. Los símbolos presentan cualidades metafóricas basadas en características compartidas por ambos móviles de comparación, puesto que las relaciones de significado se entablarían principalmente sobre la base de las múltiples cualidades de las cosas (Ricoeur 1977; Tilley 1991). Así, por ejemplo, la conformación, continuidad y extensión del simbolismo del felino en grandes áreas espaciales y por largos periodos temporales -y en sociedades tan disímiles entre sí en cuanto a su organización social- podría ser entendido, en parte, por la potencia de este animal como metáfora de ciertas actitudes humanas, debido al aspecto físico y a las cualidades de agresión y fuerza detentados por este espécimen (Saunders 1990).

Siguiendo esto, es probable entonces que las características morfológicas y las habilidades cognitivas manifestadas por las especies del género Ara, por ejemplo, haya tornado a las mismas en particularmente adecuadas para desempeñarse como fuentes de significados metafóricos para las sociedades productoras del arte mobiliar cerámico del Paraná ${ }^{10}$.

\section{Notas}

1 Sus argumentos a favor de una utilización ceremonial de las figuras representativas se afirman en criterios tales como que las proporciones alcanzadas por algunos ejemplares dificultaría su empleo como asas y que, por otra parte, la costumbre de romper las alfarerías no habría comprendido de forma deliberada a estas figuras (Torres 1911).

2 Esta suposición la respaldan en la abundancia de estas representaciones en los sitios, en la rusticidad de algunos de estos ejemplares y en los rastros de hollín que evidencian algunos de los contenedores (Aparicio 1936, 1948 ;Frenguelli 1927).

3 El cautiverio y la domesticación de psitácidos que la información etnohistórica evidencia para algunos grupos del Litoral, es tomado por tales autores como uno de los argumentos que sustentan la reiteración de estas especies en el arte alfarero.

4 Unos años más tarde Serrano (1961) modifica su postura original, sosteniendo la posibilidad de que el mundo del mito haya podido alimentar en alguna medida el mundo del arte ; aunque, sin embargo, no estos planteos no logran efectivizarse metodológicamente.

5 Estas líneas incisas no resultan exclusivas de las representaciones de psitácidos, pudiéndoselas registrar también en algunas representaciones de otros taxones (ej. diversos tipos de mamíferos). Sin embargo, su registro en estos casos se encuentra acompañado de otros rasgos característicos de las aves, como sería el diseño de probables plumas realizadas mediante incisión y modelado en la parte superior (coronilla y nuca) de las figuras, y mediante incisión en la parte lateral (correspondiente al cuello y área de inserción de las alas) de las mismas.

6 Remitirse al apartado destinado a "Los sitios arqueológicos considerados" para detalles de las excavaciones realizadas en los mismos.

7 La pintura suele ser roja, y en menor medida blanca; la cual recubre totalmente las superficies de los recipientes, forma guardas abstractas de grandes proporciones o bien conforma anchas bandas paralelas a la línea del borde.

8 Para otros datos con respecto a la discusión acerca del carácter húmedo generalizado asociado a este evento ver Tonni 2004. 
9 Vale aclarar que los análisis distribucionales pueden llegar a verse afectados como resultado de las alteraciones postdepositacionales por acción antrópica, especialmente considerables en los niveles superiores de Las Mulas 1 (ver Ceruti 1991).

10 A modo de generar problemas de estudio y de hipótesis con respecto a esto puede resultar útil la evaluación de algunas de las referencias disponibles en relación al simbolismo de las aves presentes en los registros etnohistóricos y etnográficos de poblaciones igualmente cazdoras-recolectoras, como ser el caso de los grupos Guaycurúes y Mataco-Matagallos de America del Sur (Ver Ottalagano 2007d).

\section{Bibliografía}

Aparicio, $\mathrm{F}$. 1936. El Paraná y sus tributarios. Historia de la Nación Argentina 1: 473-506. 1948. The Archaeology of the Paraná River. Bureau of American Ethnology Bulletin 3 (143): 5767.

Broecker, W.

2001. Was the Medieval Warm Period global. Science 291:1497-1499.

Campos, G.

2001. Análisis litogenético de la matriz sedimentaria de los sitios La Lechuza y Puesto Perita . Tesis de Licenciatura. Universidad Nacional de Rosario, Rosario.

Ceruti, C. N.

1980. Arroyo Arenal 4: un hallazgo arqueológico poco común . Municipalidad de La Paz, La Paz.

1988. Modificación ambiental y adaptación cultural en la cuenca del Paraná Medio. Ponencia presentada en el IX Congreso Nacional de Arqueología Argentina, Bs As.

1989. Las modificaciones ambientales del Pleistoceno final Holoceno, y su relación con los asentamientos humanos en el noroeste de Entre Ríos. PID-CONICET No 3-81800/88, Informe Final, ms.

1991. Arroyo Las Mulas I (Departamento La Paz, provincia de Entre Ríos): Relaciones hombre- medio ambiente en la actualidad y alteraciones del sitio poracción antrópica. Revista de Antropología 10: 34-45.

1999. Entidades culturales presentes en la cuenca del Paraná Medio (margen entrerriana).Mundo de Antes 3:111-135.

2000. Ríos y praderas. Los pueblos del Litoral. Nueva historia argentina (ed. por M. Tarragó), pp. 105-146. Editorial Sudamericana, Buenos Aires.

2004. Movimientos poblacionales en el Chaco Santafesino. Una visión desde la arqueología. Ponencia presentada en el Segundo Simposio sobre el estado actual del conocimiento antropológico del Gran Chaco Meridional (XXIV Encuentro de Geohistoria Regional). Argentina, Resistencia.

Chebez, J.C.

1994. Los que se van. Especies argentinas en peligro. Albatros, Buenos Aires 
Deschamps, J., O. Otero y E. Tonni

2003. Cambio climático en la pampa bonaerense: las precipitaciones desde los siglos XVIII al XX. Documento de Trabajo 109: 1-20.

Douglas, $\mathrm{M}$.

1990. The pangolin revisited: a new approach to animal symbolism. Signifying animals: Human meaning in the natural world (ed . por R. Willis), pp. 159-178.

Routledge, London.

Fiore, D.

1996. El arte rupestre como producto complejo de procesos económicos e ideológicos: una propuesta de análisis. Prehistoria y Arqueología 9: 239-259.

Foster, M.

1994. Symbolism: the foundation of culture.Companion Encyclopedia of Anthropology (ed. por T. Ingold), pp. 366-395. Routledge, Londres.

Frenguelli, J.

1927. Nuevo Tipo de Alfarería Indígena Ornitomorfa. Boletín de Educación 24:2-11.

1941. Rasgos principales de fitogeografía argentina . Coni, La Plata.

González, A. R.

1947.Investigaciones Arqueológicas en las Nacientes del Paraná Pavón. Universidad Nacional de Córdoba, Córdoba.

1977.Arte Precolombino de la Argentina. Introducción a su historia cultural. Imprenta Coni, Buenos Aires.

Handl, R.W.

2008. Aves de Chaco y Corrientes. Actualizado enero, 2008. http:/ / ntes.iespana.es (03 marzo 2008).

Hanzak, J.

1968. Gran enciclopedia ilustrada de las aves. Editorial Lectura, Caracas.

Hodder, I.

1982. The present past (An introduction to Anthropology for the Archaeologist). Pica Press, New York.

Ingold, $\mathrm{T}$.

1993. Tool -use, sociality and intelligence. Tools, Language and Cognition in Human Evolution (ed. por K. Gibson y T. Ingold) , pp. 429-445. Cambridge University Press, Cambridge.

1996. Hunting and gathering as ways of perceiving the environment. Redefining nature: ecology, culture and domestication (ed. por R. Ellen y K. Fukui) , pp. 117-155. Berg. Oxford.

Iriondo, $\mathrm{M}$.

1991. El Holoceno en el Litoral. Comunicaciones del Museo Provincial de Ciencias Naturales "Florentino Ameghino" (Nueva serie) , 3 (1): 1-39. 
Iwaniuk, A., K. Dean. y J. Nelson

2003. The evolution of the parrot brain : comparisons whit primates and other birds. Trabajo presentado al 2003 Meeting of the Society for Neuroscience. http://www.sfn.org (14 de diciembre 2007).

Levi-Strauss, C.

1997. El totemismo en la actualidad. Fondo de Cultura Económica, Bogotá.

Manzi, R. y H. Premoli.

1973. Descripción física. Flora y Fauna. Paraná, el pariente del mar (ed. por R. Naranjo, R. Vinacua, J. Riestra), pp. 17-159. Editorial Biblioteca, Rosario

Miller, D.

1994. Artifacts and the meaning of things. Companion Encyclopedia of Anthropology (ed. por T. Ingold), pp. 396-419. Routledge, Londres.

Morphy, $\mathrm{H}$.

1994. The anthropology of art. Companion Encyclopedia of Anthropology (ed. por T. Ingold),pp. 648-685. Routledge, Londres.

Narosky, T. y D. Yzurieta

2004. Birds of Argentina \& Uruguay. Vazquez Mazzini Editorial, Bs As.

Narosky, T. y P. Canevari

2004. 100 Aves argentinas. Albatros, Buenos Aires.

Ottalagano, F.

2005. Eudios cerámicos en el Nordeste Argentino: algunos datos acerca de las manifestaciones simbólico-iconográficas paranaenses. Trabajo presentado al XI Congreso Nacional de Arqueología Uruguaya, Salto.

2007 a. Algunos datos sobre las manifestaciones simbólico-iconográficas de las costas del Paraná: estudios cerámicos en Las Mulas 1 (Provincia de Entre Ríos). Arqueología en las Pampas (ed.por C. Bayón, A. Pupio, M.I. González, N. Flegenheimer y M, Frére), 679-696. Sociedad Argentina de antropología , Bs. As.

2007 b. Propuesta e implementación de medidas metodológicas para el estudio de los motivos abstractos y representativos cerámicos de Arroyo Arenal 1 (Dpto. La Paz, Pcia de Entre Ríos).Revista de la Escuela de Antropología 13. Humanidades y Artes Ediciones, Rosario.

2007c. Una propuesta de abordaje para el estudio del arte mobiliar cerámico de cazadoresrecolectores del litoral fluvial del Paraná. Ponencia presentada al XVI Congreso Nacional de Arqueología. Argentina, Jujuy.

2007d. Algunas referencias en torno al simbolismo de las aves en los registros etnohistóricos y entnográficos de guaycurúes y mataco-mataguayos. Arqueología Suramericana 3(2): 213228.

Parodi, L.

1964. Las regiones fitogeográficas de la Argentina. Enciclopedia de agricultura y jardinería (ed. por L. Parodi ), pp. 1-14. Editorial Acme, Bs. As. 
Renfrew, C.

1994. Toward a Cognitive Archaeology. The ancient mind: element of Cognitive Archaeology (ed. por C. Renfrew y E. Zubrow ), pp. 3-12. Cambridge University Press, Cambridge.

Ricoeur, P.

1977. La metáfora viva. Megalópolis, Buenos Aires.

Ringuelet, R.

1961. Rasgos fundamentales de la zoogeografía de la Argentina. Physis 22 (63): 151-170.

Salemme, M., E. Tonni, C. Ceruti, M. Iriondo y A.Cione

1987. Los vertebrados del sitio arqueológico La Palmera V , Departamento Paraná, Pcia. de Entre Ríos. Ponencia. III Jornadas de Ciencias Naturales del Litoral, Argentina, Corrientes.

Saunders, N.

1990. Tezcatlipoca: jaguar metaphors and the Aztec mirror of nature. Signifying animals. Human meaning in the natural World (ed. por R. Willis) , pp. 159-178. Routledge, London.

Serrano, A.

1921.Contribución al conocimiento de la Arqueología de los clrededores de Paraná, Paraná.1946. Arqueología del Arroyo las Mulas en el noroeste de Entre Ríos. Universidad de Córdoba, Córdoba. 1961. El Arte Plástico de los Ribereños Paranaenses. Nordeste 2:73-86.

Spinuzza, J.

2008. Aves de la llanura pampeana. 18 marzo, 2008. http://www.avespampa.com.ar / Psittaciformes.htm (21 marzo 2008).

Strahler, A.

1975. Geografía física. Ediciones Omega, Barcelona.

Tapper, Richard

1994. Animality, humanity, morality, society. What is an animal? (ed. por T. Ingold) , pp. 4762. Routledge, London

Tilley, C.

1991. Material Culture and text: the art of ambiguity.Routledge, Londres.

Tonni, E.

2004. Faunas y climas del cuaternario en la Mesopotamia Argentina. Miscelánea 12: 31-38.

Tonni, E., C. Ceruti y M. Iriondo

1985. Los vertebrados del sitio Arenal, Departamento La Paz, Provincia de Entre Ríos (Argentina). Revista de la asociación de ciencias naturales del Litoral 16 (2): 157-167.

Torres, L.M.

1911. El totemismo. Su origen, Significado y Supervivencias. Anales del Museo de Buenos Aires 20: 485-553. 
Vigil, C.

1973. Aves argentinas y sudamericanas. Atlántida, Buenos Aires. 